\title{
SALINIDADE NA GERMINAÇÃO DE SEMENTES DE MADEIRA-NOVA INDUZIDA POR DIFERENTES SAIS
}

\section{SALINIDAD EN LA ALEMINACIÓN DE SEMILLAS DE MADEIRA-NOVA INDUCIDA POR DIFERENTES SALES}

\section{SALINITY IN GERMINATION OF MADEIRA-NOVA SEED INDUCED BY DIFFERENT SALTS}

Emanuelle Marques Coutinho ${ }^{1}$; Andrei dos Santos Souza ${ }^{2}$; Mateus Pereira dos Santos ${ }^{3}$; Jamil Sousa Silva ${ }^{4}$; Gisele Brito Rodrigues ${ }^{5}$

DOI: https://doi.org/10.31692/978-65-991061-4-9.192-198

\section{INTRODUÇÃO}

A Madeira-nova (Pterogyne nitens Tul.) se destaca entre as espécies nativas mais cultivadas no Brasil, sendo de grande importância econômica para a produção madeireira e geração de energia, bem como tem sido muito utilizada na reposição de matas ciliares e recuperação de vegetação (FIGUEIREDO et al., 2018). A produção de mudas requerida na recuperação de áreas degradas é dificultada em decorrência da dormência das sementes desta espécie, dificultando a sua germinação.

Para germinar, além de superar a dormência, a espécie requer condições de luz, temperatura e disponibilidade de água. Em condições de solos com alta concentração salina, as sementes apresentam dificuldades para germinar, visto que o alto teor de sais, especialmente de cloreto de sódio $(\mathrm{NaCl})$, pode inibir a germinação devido a diminuição do potencial osmótico, ocasionando prejuízos as demais fases do processo. Além disso, o sal absorvido, acumulado no interior das células, pode causar toxidez (AVRELLA, 2019).

Segundo Santos (2011), a salinidade é um fator abiótico que afeta negativamente o processo de germinação, o desenvolvimento vegetativo das culturas, e nos casos mais graves, leva a morte das plântulas. Portanto, torna-se necessário obter informações sobre o efeito da salinidade no processo germinativo, a fim de possibilitá-lo nessas condições. Assim, o objetivo desse trabalho foi avaliar o efeito de diferentes potencias osmóticos induzidos pelos

\footnotetext{
1 Curso de graduação em Agronomia, Universidade Estadual do Sudoeste da Bahia - UESB, e-mail: emanuellemarques321@gmail.com

2 Curso de graduação em Agronomia, Universidade Estadual do Sudoeste da Bahia - UESB, e-mail: andrei_souza37@hotmail.com

3 Curso de graduação em Agronomia, Universidade Estadual do Sudoeste da Bahia - UESB, e-mail: mateus.santos.0712@gmail.com

4 Curso de graduação em Agronomia, Universidade Estadual do Sudoeste da Bahia - UESB, e-mail: jamilsousa13@gmail.com

${ }^{5}$ Doutora em Agronomia (Fitotecnia)/Departamento de Fitotecnia e Zootecnia da UESB, Universidade Estadual do Sudoeste da Bahia - UESB, e-mail: gisele.rodrigues@uesb.edu.br
} 
agente salinos $\mathrm{CaCl}_{2} 2 \mathrm{H}_{2} \mathrm{O}$ e $\mathrm{NaCl}$ na germinação de sementes de Pterogyne nitens.

\section{FUNDAMENTAÇÃO TEÓRICA}

A busca pelo entendimento sobre a capacidade de alguns vegetais em tolerar condições de baixa disponibilidade hídrica e a caracterização dos problemas de ordem fisiológica ou ecológica causada pela escassez de água merecem significativa importância para o meio científico, de forma que sejam geradas tecnologias que permita a exploração dessas espécies, sobretudo para a sua utilização em recuperação de locais onde ocorre esse tipo de limitação (SÁ et al., 2013).

A madeira-nova pertence à família Fabaceae (Subfamília Caesalpinoideae) e apresenta características importantes como rusticidade e crescimento rápido, por esta razão, é muito utilizada em plantio para recuperação de áreas degradadas e de preservação permanente. Além disso, possui ampla distribuição natural por todo o Brasil, ocorrendo em biomas como Mata Atlântica, Cerrado, Caatinga, em também em áreas caracterizadas pela elevada umidade e vegetação florestal (LORENZI, 2009). Contudo, um dos principais problemas para a propagação dessa espécie é a dormência apresentada pelas suas sementes, causando germinação lenta e desuniforme.

Para que ocorra a germinação, a disponibilidade de água se torna um fator limitante, e nessas condições o potencial germinativo de sementes de determinadas espécies podem indicar se a mesma apresentará tolerância à deficiência hídrica ou salinidade em estádios subsequentes do seu desenvolvimento (TAIZ; ZEIGER, 2013). Deste modo, há importância do estudo dos efeitos da condição de estresse ocasionado pela salinidade no processo germinativo de algumas espécies.

Ressalta-se que, quando este tipo de estresse ocorre de forma severa na planta-mãe, os efeitos refletem de forma indireta na germinação das sementes produzidas, pois estas, quando submetidas à potenciais osmóticos muitos negativos, não conseguirão absorver água, tendo o processo germinativo e a velocidade de germinação prejudicados, além disso, a plântula formada tende a apresentar menor resistência as condições ambientais adversas e de fatores bióticos como o ao ataque de patógenos e pragas (KAPPES et al., 2010).

\section{METODOLOGIA}

O experimento foi conduzido no Laboratório de Tecnologia e Produção de Sementes da Universidade Estadual do Sudoeste da Bahia - UESB, Campus Vitória da Conquista, BA (1450'19" S e 4450'19"W). Foram coletadas frutos de Madeira-nova em diferentes matrizes 
localizadas no referido campus. Após as coletas, as sementes foram extraídas manualmente dos frutos com o auxílio de uma tesoura e selecionadas quanto à integridade física e ausência de ataques de patógenos e insetos.

O delineamento experimental utilizado foi o inteiramente casualizado em esquema fatorial $5 \times 2$, composto por cinco potenciais osmóticos $(-0,1 ;-0,2 ;-0,3 ;-0,4$ e $-0,5 \mathrm{MPa})$ e dois agentes salinizantes (Cloreto de Cálcio Dihidratado - $\mathrm{CaCl}_{2} 2 \mathrm{H}_{2} \mathrm{O}$ e Cloreto de Sódio - $\mathrm{NaCl}$ ), com quatro repetições de 25 sementes, totalizando 40 parcelas. As soluções foram preparadas nas concentrações de cada potencial indicado seguindo a fórmula de Van’t Hoff:

$$
\Psi_{\text {osm }}=-\mathrm{RTC}
$$

Onde: $\Psi_{\text {osm }}$ é o potencial osmótico (atm); R é a constante geral dos gases $=0,082 \mathrm{~atm}$ $\mathrm{L} \mathrm{mol}^{-1}{ }^{\circ} \mathrm{K}^{-1}$; T é a temperatura $\left({ }^{\circ} \mathrm{K}\right)$ e $\mathrm{C}$ a concentração molar (mols de soluto $10^{-3} \mathrm{~g}^{-1} \mathrm{de}$ água).

As sementes de madeira-nova, antes dos testes, foram submetidas à superação de dormência através de escarificação química com Ácido Sulfúrico $\left(\mathrm{H}_{2} \mathrm{SO}_{4}\right)$, onde ficaram imersas por 10 minutos, sendo logo em seguida, desinfestadas com Hipoclorito de sódio à $1,5 \%$.

As sementes foram distribuídas uniformemente sobre uma folha de papel Germitest ${ }^{\circledR}$ e foram cobertas por outra folha. Esses papeis foram umedecidos com as soluções salinas na proporção de 2,5 vezes o peso do papel seco, segundo a metodologia padrão para teste de germinação das Regras para Análise de Sementes (BRASIL, 2009). Os papeis foram organizados em rolos acondicionados em sacos plásticos e mantidos em câmara B.O.D regulada à $25 \pm 0,3{ }^{\circ} \mathrm{C}$. A contagem das sementes germinadas foi feita diariamente, considerando germinadas as sementes que apresentaram a protrusão da radícula maior ou igual a 5 mm (BRASIL, 2009).

Avaliou-se a Porcentagem de Germinação (\%), o Índice de Velocidade de Germinação (IVG), o Tempo Médio de Germinação (dias), a Velocidade Média de Germinação $\left(\operatorname{dias}^{-1}\right)$, o Índice de Sincronização de Germinação ou incerteza (bits) e o Coeficiente de Uniformidade de Germinação. As variáveis foram analisadas pelo pacote germinationmetrics do Programa livre R (R Development Core Team, 2016) e em seguida, os dados foram submetidos à análise de variância e regressão, sendo comparados pelo teste Tukey à 5\% de probabilidade pelo mesmo programa através do pacote Expdes.pt.

\section{RESULTADOS E DISCUSSÃO}


Os resultados da análise de variância indicaram interação significativa entre os potenciais osmóticos e os agentes salinizantes (Tabela 1).

Tabela 1. Médias de Germinação (\%), Índice de Velocidade de Germinação (IVG), Tempo Médio e Velocidade Média de Germinação (TMG), (VMG), Índice de Sincronização de germinação (E) e Coeficiente de Uniformidade de Germinação (CUG) de sementes de $P$. nitens submetidas à estresse salino induzido por

\begin{tabular}{|c|c|c|c|c|c|c|}
\hline \multirow{2}{*}{ Agente osmótico } & Germinação (\%) & IVG & TMG (dias) & VMG $\left(\operatorname{dias}^{-1}\right)$ & $\mathrm{E}$ (bits) & CUG \\
\hline & \multicolumn{6}{|c|}{$-0,1 \mathrm{MPa}$} \\
\hline $\mathrm{CaCl}_{2} 2 \mathrm{H}_{2} \mathrm{O}$ & $63,71 \mathrm{~B}$ & $5,36 \mathrm{~B}$ & $3,98 \mathrm{~A}$ & $0,25 \mathrm{~B}$ & $0,37 \mathrm{~A}$ & $0,28 \mathrm{~A}$ \\
\hline \multirow[t]{2}{*}{$\mathrm{NaCl}$} & $74,22 \mathrm{~A}$ & $9,83 \mathrm{~A}$ & $2,59 \mathrm{~B}$ & $0,38 \mathrm{~A}$ & $0,48 \mathrm{~A}$ & $0,14 \mathrm{~B}$ \\
\hline & \multicolumn{6}{|c|}{$-0,2 \mathrm{MPa}$} \\
\hline $\mathrm{CaCl}_{2} 2 \mathrm{H}_{2} \mathrm{O}$ & $62,23 \mathrm{~A}$ & $4,22 \mathrm{~B}$ & $4,72 \mathrm{~A}$ & $0,21 \mathrm{~B}$ & $0,39 \mathrm{~B}$ & $0,47 \mathrm{~A}$ \\
\hline \multirow[t]{2}{*}{$\mathrm{NaCl}$} & $66,76 \mathrm{~A}$ & $9,44 \mathrm{~A}$ & $2,38 \mathrm{~B}$ & $0,42 \mathrm{~A}$ & $0,66 \mathrm{~A}$ & $0,13 \mathrm{~B}$ \\
\hline & \multicolumn{6}{|c|}{$-0,3 \mathrm{MPa}$} \\
\hline $\mathrm{CaCl}_{2} 2 \mathrm{H}_{2} \mathrm{O}$ & $0,00 \mathrm{~B}$ & $0,00 \mathrm{~B}$ & $0,00 \mathrm{~B}$ & $0,00 \mathrm{~B}$ & $0,00 \mathrm{~B}$ & $0,00 \mathrm{~B}$ \\
\hline \multirow[t]{2}{*}{$\mathrm{NaCl}$} & $61,35 \mathrm{~A}$ & $6,91 \mathrm{~A}$ & $2,98 \mathrm{~A}$ & $0,33 \mathrm{~A}$ & $0,56 \mathrm{~A}$ & $0,16 \mathrm{~A}$ \\
\hline & \multicolumn{6}{|c|}{$-0,4 \mathrm{MPa}$} \\
\hline $\mathrm{CaCl}_{2} 2 \mathrm{H}_{2} \mathrm{O}$ & $0,00 \mathrm{~B}$ & $0,00 \mathrm{~B}$ & $0,00 \mathrm{~B}$ & $0,00 \mathrm{~B}$ & $0,00 \mathrm{~B}$ & $0,00 \mathrm{~B}$ \\
\hline \multirow[t]{2}{*}{$\mathrm{NaCl}$} & $63,23 \mathrm{~A}$ & $8,59 \mathrm{~A}$ & $2,44 \mathrm{~A}$ & $0,41 \mathrm{~A}$ & $0,57 \mathrm{~A}$ & $0,13 \mathrm{~A}$ \\
\hline & \multicolumn{6}{|c|}{$-0,5 \mathrm{MPa}$} \\
\hline $\mathrm{CaCl}_{2} 2 \mathrm{H}_{2} \mathrm{O}$ & $0,00 \mathrm{~B}$ & $0,00 \mathrm{~B}$ & $0,00 \mathrm{~B}$ & $0,00 \mathrm{~B}$ & $0,00 \mathrm{~B}$ & $0,00 \mathrm{~B}$ \\
\hline $\mathrm{NaCl}$ & $60,86 \mathrm{~A}$ & $4,84 \mathrm{~A}$ & $4,23 \mathrm{~A}$ & $0,23 \mathrm{~A}$ & $0,34 \mathrm{~A}$ & $0,32 \mathrm{~A}$ \\
\hline
\end{tabular}

Médias seguidas de mesma letra maiúscula na coluna, não diferem entre si pelo teste Tukey à 5\% de probabilidade.

Fonte: Própria (2019)

As médias de Germinação e IVG com $\mathrm{NaCl}$ foram superiores às médias das mesmas variáveis para os tratamentos com $\mathrm{CaCl}_{2} 2 \mathrm{H}_{2} \mathrm{O}$ dentro de todos os potenciais osmóticos, exceto -0,2 MPa, onde as médias de Germinação não diferiram entre si. Verifica-se que, para $\mathrm{CaCl}_{2} 2 \mathrm{H}_{2} \mathrm{O}$, a germinação foi nula a partir $-0,3 \mathrm{MPa}$.

O mesmo comportamento foi observado por Santos et al. (2016) para as espécies Poincianela pyramidalis (Tul.) L. P. Queiroz e Anadenanthera colubrina (Vell.), os autores observaram que a germinação e o IVG dessas espécies sob solução de $\mathrm{CaCl}_{2}$ foi nula a partir de -0,8 MPa, possivelmente devido alterações na concentração dos íons $\mathrm{Na}^{+}$e $\mathrm{Cl}^{-}$no interior das células, ocasionando estresse iônico e descontrole em algumas atividades metabólicas durante o processo de germinação.

Os valores de TMG foram maiores nos tratamentos $\mathrm{CaCl}_{2} 2 \mathrm{H}_{2} \mathrm{O}$, nos potenciais de 0,1 e -0,2 $\mathrm{MPa}$ em relação aos tratamentos com $\mathrm{NaCl}$, indicando atraso na germinação das sementes em contato com esse sal, o que já era esperado. Ressalta-se que, os valores nulos de 
TMG obtidos nos potenciais de 0,$3 ;-0,4$ e - 0,5 MPa foram em decorrência da não germinação das sementes, por isso, as médias dos tratamentos com $\mathrm{NaCl}$ foram superiores nos referidos potenciais. Já para a VMG, que é inversamente proporcional ao TMG, maiores médias foram obtidas com $\mathrm{NaCl}$ em todos os potenciais osmóticos quando comparados com $\mathrm{CaCl}_{2} 2 \mathrm{H}_{2} \mathrm{O}$, evidenciando que as sementes apresentaram germinação mais veloz quando tratadas com $\mathrm{NaCl}$.

Os tratamentos com $\mathrm{CaCl}_{2} 2 \mathrm{H}_{2} \mathrm{O}$ apresentaram germinação mais sincronizada do que os tratamentos com $\mathrm{NaCl}$, devido ao menor valor E encontrado, já para o CUG, verificou-se baixos valores para esta variável, indicando germinação desuniforme em todos os tratamentos, possivelmente esses resultados ocorreram em função da alta concentração dos íons $\mathrm{Na}^{+} \mathrm{e} \mathrm{Cl}^{-}$ no interior celular que foi suficiente para provocar respostas diferentes quanto à uniformização e sincronia da germinação.

A análise de regressão indicou efeito linear negativo para todas as variáveis dos tratamentos com $\mathrm{CaCl}_{2} 2 \mathrm{H}_{2} \mathrm{O}$, ou seja, a medida que o potencial osmótico é reduzido, as características da germinação também se reduzem (Figura 1). Já para os tratamentos com $\mathrm{NaCl}$, foi verificado efeito linear negativo para Germinação e IVG, efeito linear positivo para TMG, efeito quadrático positivo para VMG e Índice de sincronização (E) e quadrático negativo para CUG (Figura 1).

Figura 1. Estimativa da Germinação (\%) (A), Índice de Velocidade de Germinação (IVG) (B), Tempo Médio de Germinação (TMG) (dias) (C), Velocidade Média de Germinação (VMG) (dias ${ }^{-1}$ ) (D), Índice de Sincronização de Germinação (E) (bits) (E) e Coeficiente de Uniformidade de Germinação (CUG) (F) de sementes de $P$. nitens submetidas à salinidade $\mathrm{com} \mathrm{CaCl}_{2}$ $2 \mathrm{H}_{2} \mathrm{O}$ e NaCl, Vitória da Conquista, BA, 2019. * e ** significativo à 1 e $5 \%$ de probabilidade.
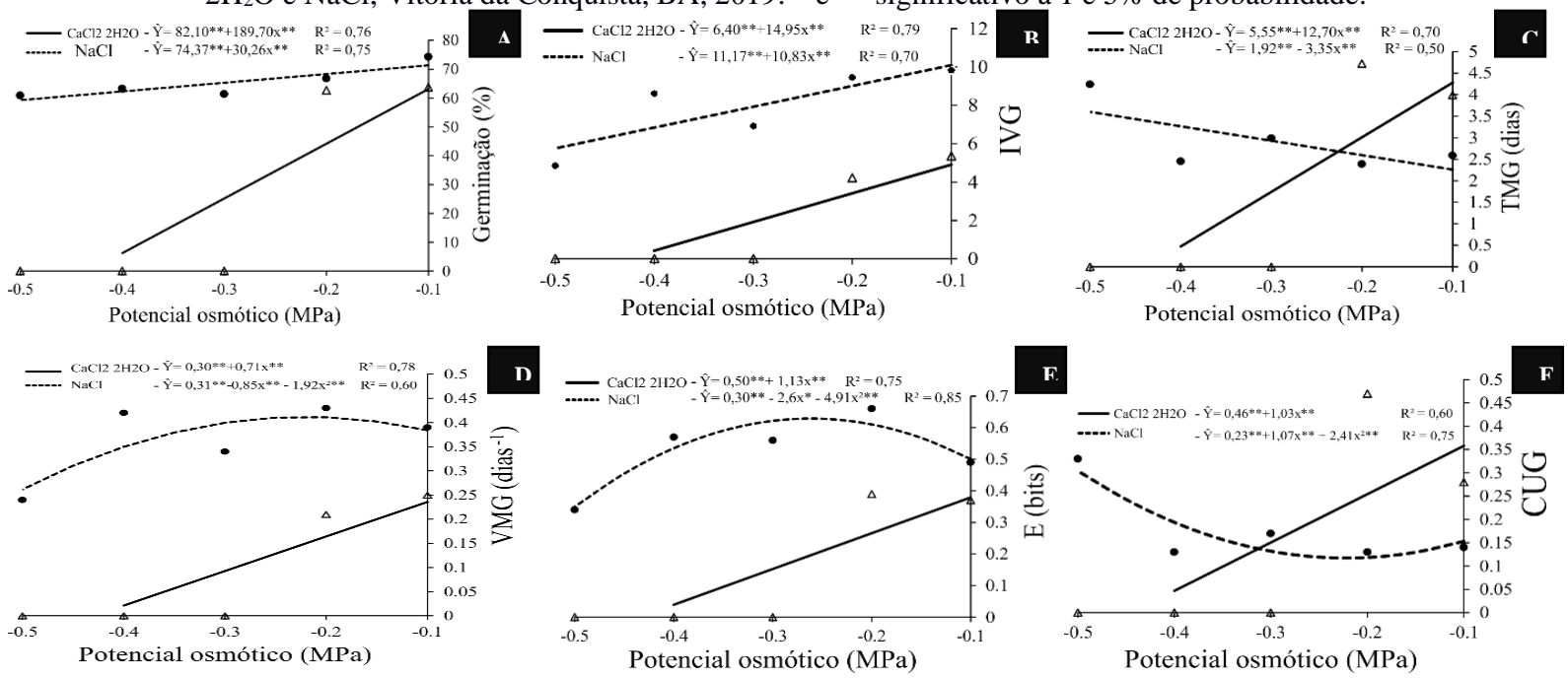

Os pontos de máxima de VMG foi de -0,22 $\mathrm{MPa}$, atingindo o valor de 0,41 dias ${ }^{-1}$ depois desse valor a VMG decresce. Para o Índice de sincronização o ponto de máxima foi de -0,26 MPa, alcançando valor máximo de 0,62 bits decrescendo a partir desse ponto. Para 
CUG, o ponto de mínima atingindo foi -0,22 MPa resultando num valor de 0,11 e aumentado a partir daí.

Esses resultados corroboram com os obtidos por Dutra et al. (2017), os autores encontraram efeitos lineares negativos para quase todas as variáveis avaliadas, principalmente a porcentagem de germinação. Esses resultados indicam que essa espécie apresenta pouca tolerância à salinidade induzida por $\mathrm{CaCl}_{2} 2 \mathrm{H}_{2} \mathrm{O}$, de forma que, a sua germinação pode ser prejudicada caso seja submetida à solos com alto teor de cálcio e cloro com potenciais osmóticos acima de -0,3 MPa. Em contrapartida, a Madeira-nova apresentou potencial germinativo tolerante ao $\mathrm{NaCl}$, contudo, em concentrações de potencial osmótico não muito negativos, já que a tendência de germinação dessa espécie é reduzir com o aumento da concentração salina.

\section{CONCLUSÃO}

A germinação de sementes de Madeira-nova é severamente reduzida em potenciais osmóticos induzidos por $\mathrm{CaCl}_{2} 2 \mathrm{H}_{2} \mathrm{O}$ e pouco prejudicada por $\mathrm{NaCl}$, indicando que a germinação das sementes dessa espécie apresenta certa tolerância à esse tipo de sal.

\section{REFERÊNCIAS}

AVREllA, E. D., EMER, A. A., PAIM, L. P., FIOR, C. S., \& SCHAFER, G. Efeito da salinidade no desenvolvimento inicial de mudas de Mimosa scabrella Benth. Iheringia. Série Botânica. v. 74, 2019.

Brasil. Ministério da Agricultura e Reforma Agrária. Secretária de Defesa Agropecuária. Regras para análise de sementes. Brasília, DF, 2009. 399 p.

DUTRA, T. R.; MASSAD, M.D.; MOREIRA, P.R.; RIBEIRO, E.S.M. Efeito da salinidade na germinação e crescimento inicial de plântulas de três espécies arbóreas florestais. Pesquisa Florestal Brasileira, v. 37, n. 91, p. 323-330, 2017.

FIGUEIREDO, M. E. O., LONGUE JÚNIOR, D., PEREIRA, A. K. S., CARNEIRO, A. D. C. O., SILVA, C. M. S. D. Potential of Pterogyne nitens tul. wood (Madeira-nova) for charcoal production. Ciência Florestal, v. 28, n. 1, p. 420-431, 2018.

KAPPES, C. et al. Germinação, vigor de sementes e crescimento de plântulas de milho sob condições de déficit hídrico. Scientia Agraria, Piracicaba, v.11, n.2, p.125-134, 2010.

LORENZI, H. Árvores brasileiras: Manual de identificação e cultivo de plantas arbóreas nativas do Brasil. Nova Odessa: Plantarum, 2009. p. 197.

R Core Team. R: a language and environment for statistical computing. Vienna: R Foundation for Statistical Computing, 2015. Disponível em:< http://www.R-project.org/.>. Acesso em: 01 
ago. 2019.

SÁ, F. V. S. et al. Crescimento inicial de arbóreas nativas em solo salino-sódico do Nordeste brasileiro tratado com corretivos. Revista Ceres, Viçosa, v. 60, n. 3, p. 388-396, 2013.

SANTOS, A. R.; SILVA-MANN, R.; FERREIRA, R. A. Restrição hídrica em sementes de jenipapo (Genipa americana L.). Revista Árvore, Viçosa, v.35, n.2, p.213-220, 2011.

SANTOS, C.A. dos; SILVA, N.V da; WALTER, L.S; SILVA, E.C.A da; NOGUEIRA, R.J.M.C. Germinação de duas espécies da caatinga sob déficit hídrico e salinidade. Pesquisa Florestal Brasileira, v. 36, n. 87, p. 219-224, 2016.

TAIZ, L.; ZEIGER, E. Fisiologia vegetal. 5.ed. Porto Alegre: Artmed, 2013. 954 p. 